\title{
Airway obstruction and rheumatoid arthritis
}

\author{
A. Vergnenègre*, N. Pugnere**, M.T. Antonini+, M. Arnaud ${ }^{++}$, B. Melloni**, \\ R. Treves,++ F. Bonnaud**
}

Airway obstruction and rheumatoid arthritis. A. Vergnenègre, N. Pugnere, M.T. Antonini, M. Arnaud, B. Melloni, R. Treves, F. Bonnaud. @ERS Journals Ltd 1997.

ABSTRACT: The aim of this study was to assess the percentage of respiratory disorders and airway obstruction in patients with rheumatoid arthritis by comparing lung function test results between patients with rheumatoid arthritis and control subjects with other rheumatological conditions.

A prospective case-control study of respiratory symptoms and lung function abnormalities was performed in a series of 100 patients with rheumatoid arthritis. Eighty eight patients with other rheumatological diseases served as controls. Diagnosis of respiratory disorders was based on clinical, radiological and spirometric findings. Airway obstruction was determined from predicted values. The results were compared using Student's t-test and Chi-squared tests. An explanatory analysis was carried out by linear regression.

The number of symptoms, respiratory disorders (including bronchiectasis) and lung function abnormalities was higher in patients with rheumatoid arthritis than in controls. After exclusion of smokers, the proportion of airway obstruction in patients with rheumatoid arthritis was $16 \%$ (versus $0 \%$ in controls), although the patients with rheumatoid arthritis still had more symptoms and respiratory disorders. The Chi-squared test did not identify any relationship between airway obstruction, duration of rheumatoid arthritis and type of treatment and occurrence of Sjögren's syndrome. In the nonsmokers, different explanatory models for the patients with and without a history of cardiac and respiratory disease emerged from multivariate analysis of indices of obstruction. These models included variables characterizing the severity and course of the rheumatoid arthritis.

Respiratory disorders (including bronchiectasis) and airway obstruction are more frequent among patients with rheumatoid arthritis than in rheumatological controls. Although the exact pathophysiology of the link between bronchial obstruction and rheumatoid arthritis is still a matter of debate, explanatory factors for obstruction included variables characterizing the rheumatoid arthritis. Eur Respir J 1997; 10: 1072-1078.

\begin{abstract}
* Medical Information Unit, Chest Disease Dept, **Chest Disease Dept, +Pulmonary Function Test Laboratory, and ${ }^{++}$Rheumatologic Unit, University Hospital, Limoges, France.
\end{abstract}

Correspondence: A. Vergnenègre

Unité d'Information Médicale

Service de Pneumologie

Hôpital Universitaire du Cluzeau

87042 Limoges

France

Keywords: Airway obstruction

bronchiectasis

epidemiology

rheumatoid arthritis

Received: March 251996

Accepted after revision January 161997
A large number of studies have been devoted to pulmonary disorders in patients with rheumatoid arthritis (RA). The well-characterized disorders include: pleural effusions; rheumatoid nodules; pulmonary fibrosis; and Caplan's syndrome $[1,2]$. Bronchiectasis and an increased incidence of chest infections have also been reported $[3,4]$, but the pathophysiology is largely unknown. Although abnormal airway function has been described in $30-40 \%$ of RA patients depending on the study [5-11], the existence of a specific airway obstruction is a subject of debate [12].

The prevalence of abnormalities might be influenced by: 1) patient selection (if they were affected by interstitial lung disease, the incidence of abnormal pulmonary function test [9] could increase to $30-40 \%$ ); 2) smoking habits [5]; 3) the methods used to demonstrate abnormal lung function: maximal mid-expiratory flow rate (MMEFR) [5, 6], maximum expiratory flow at 50\% forced vital capacity $\left(V^{\prime} \max 50\right)$ [8]. Unfortunately, there is, as yet, no universally agreed definition of what constitutes an abnormal result [11].
Among the case-control studies, some have compared pulmonary function between RA patients and controls with degenerative joint disease $[5,8,13]$, although, in most of the studies, RA patients have been compared with controls in the general population $[6,7,10,11]$.

In the present study, we attempted to eliminate the smoking effect and we employed a standardized definition of lung function abnormalities [14]. The study was designed to assess the percentage of respiratory disorders and airway obstruction in RA patients by comparing lung function between RA patients and those with other rheumatological diseases.

\section{Methods}

\section{Study population}

Between January and December 1994, 100 consecutive patients with rheumatoid arthritis, as defined by the criteria of the American Rheumatoid Association (1987), 
gave their informed consent to participate in the study. They included both new and follow-up cases attending the rheumatism unit at the Limoges University Hospital. During the same period, control data were obtained from patients attending the same unit for degenerative joint diseases or infectious rheumatological diseases. They were matched for age and sex. After giving their consent, their responses to the questionnaire were recorded and they were subjected to the same spirographic measurements as the RA patients. All patients (cases and controls) benefited from a chest radiograph.

\section{Questionnaire}

The respiratory questionnaire was based on the British Medical Research Council (BMRC) guidelines. The French version was drawn up by the World Health Organization (WHO) [15], and administered by a physician who saw the patient in the rheumatological unit.

Further questions were added about the rheumatoid disease: age of onset of RA; duration of arthritis; Ritchie's index [16]; and erythrocyte sedimentation rate (ESR). Ritchie's index is the index of reference, used for the validation of new tools in evaluation of RA patients [17]. ESR was recorded routinely in the Rheumatology Department. Sjögren's syndrome was assessed using European criteria [18], based on clinical, radiological and histological findings. Presence or absence of rheumatoid factor (with latex and Waaler Rose titration), antirheumatic medication (corticosteroids, gold, methotrexate, penicillamine, sulphasalazine, azathioprine, hydroxychloroquine) were recorded. The questionnaire was administered before the lung function examination.

Respiratory history was defined as: history of pulmonary surgery, pleurisy, pneumonia, tuberculosis, hay fever or other infrequent and nonobstructive disease. Cardiac history was extracted from the questionnaire.

\section{Definition of respiratory disorders associated with $R A$}

Chronic bronchitis was defined by cough and expectoration 3 months a year for two consecutive years, according to the criteria of the American Thoracic Society (ATS) (1960). Smoking habits were classified as: nonsmokers (who had never smoked); current smokers; or ex-smokers (abstinence for the previous 3 months). The amount smoked annually in pack-years was recorded for the smoking group; in view of the small sample size, the smoking group included both current and ex-smokers.

Asthma was defined by respiratory symptoms and spirographic findings. Fibrosis was defined by symptoms, interstitial syndrome on chest radiograph image, and restrictive syndrome (decreased total lung capacity). Bronchiectasis was suspected on clinical symptoms (cough, expectoration, coarse crackles) and radiological findings. In each of these cases, a computed tomography (CT) scan was performed. The diagnosis was only retained if it was backed-up by the tomodensitometric findings.

\section{Pulmonary function tests (PFTs)}

PFTs were performed in the same laboratory and using the same equipment. The spirometric measurements were made on a pneumotachograph (Medical Graphics 1070, St. Paul, MN, USA). At least three reproducible measurements were performed on all individuals by operators experienced with this instrument. Forced vital capacity (FVC), forced expiratory volume in one second (FEV1), and forced expiratory flow rate between 25 and $75 \%$ of vital capacity (FEF25-75) were recorded. FEV1/FVC and FEF25-75/FVC were calculated from these values. The best of the three curves was selected. Curves with poor reproducibility or of bad quality were rejected. Values were expressed as a percentage of the normal values of the European Coal and Steel Community (ECCS 1993), which are calculated according to the age, sex and height of the individual [14].

Residual volume (RV) and total lung capacity (TLC) were measured by plethysmography (Medical Graphics 1085). Single-breath transfer factor of the lung for carbon monoxide $(T \mathrm{~L}, \mathrm{CO})$ was determined by the inspiratory apnoea method (Medical Graphics 1070).

The lower limit for distal airway obstruction was a value of FEF25-75 of $1.71 \mathrm{~L} \cdot \mathrm{s}^{-1}$ less than normal in males and $1.40 \mathrm{~L} \cdot \mathrm{s}^{-1}$ less than normal in females $(1.64 \mathrm{SD})$ [14]. Distal airway obstruction was considered to be present if values were under these thresholds. The lower limit for overall airway obstruction was: normal value FEV1/ FVC minus $11.8 \%$ in males, and minus $10.7 \%$ in females (1.64 SD) [14]. Overall airway obstruction was considered to be present if values were under these thresholds. The lower limit for definition of restrictive pulmonary function was: TLC minus $1.15 \mathrm{~L}$ in males and minus $0.99 \mathrm{~L}$ in females (1.64 SD). Restrictive lung function was considered to be present if values were under these thresholds. The threshold for TL,CO was calculated in the same way.

\section{Statistical analysis}

Frequency distribution, means and SD were calculated using Statistical Products and Service Solutions (SPSS) software (Chigaco Inc.). The quantitative variables were compared using Student's t-test for unpaired data. Symptoms, previous chest and cardiac illness, and qualitative lung function results were compared using the Chi-squared test. Among lung function results, FEF25-75, FEV1/FVC were subjected to an explanatory analysis by stepwise multilinear regression (SPSS software). To introduce a variable, the p-value was 0.2 , to eliminate a variable the p-value was 0.3

\section{Results}

\section{Patient details}

During 1994, 100 hospital patients with recognized RA were included in the study. During the same period, 88 control patients (with degenerative or infectious rheumatological conditions) were also recruited for the study. Both groups gave their consent to respond to the questionnaire and, when possible, to carry out PFTs. All PFTs were analysable in the control group, against 85 in the RA group. There was no difference in demographic variables, smoking history, symptoms and treatment administered between these 85 patients and the 15 patients with no PFT results. 
The characteristics of the patients with RA and the controls are listed in table 1 . There were no statistically significant differences in demographic details, cardiac or respiratory history or smoking history. In the RA patients, respiratory history $(n=22)$ included the following: 6 pneumonia, 6 pleurisy, 1 tuberculosis, 1 thoracic surgery, 2 allergic rhinitis, 6 with various chest conditions. In the control group $(n=12), 2$ had a history of pneumonia, 1 pleurisy, 8 allergic rhinitis, and 1 another chest condition.

\section{Overall analysis of both groups $(n=188)$}

It can be seen from tables 2 and 3 that there were significant differences in functional symptoms between the two groups of patients. There were more associated respiratory disorders in the RA group $(n=26): 13$ chronic bronchitis, 5 asthma, 2 fibrosis and 6 bronchiectasis. In the control group $(n=7)$, there were 5 chronic bronchitis and 2 asthmas. There was also a significant difference for the bronchiectasis.

For the functional respiratory disorders, there was a significant difference in the indices of obstruction (table 2).

Table 1. - Characteristics of the patient with rheumatoid arthritis and of the controls

\begin{tabular}{lccc}
\hline & $\begin{array}{c}\text { Cases } \\
(\mathrm{n}=100)\end{array}$ & $\begin{array}{c}\text { Controls } \\
(\mathrm{n}=88)\end{array}$ & p-value ${ }^{\#}$ \\
\hline Age yrs $\ddagger$ & $60 \pm 12$ & $57 \pm 14$ & $\mathrm{NS}$ \\
Sex M/F & $77 / 23$ & $67 / 21$ & NS \\
BMI kg·m-2 & $24.4 \pm 3.9$ & $24.3 \pm 4.0$ & NS \\
Previous cardiac illness n & 22 & 21 & NS \\
Previous chest illness n & 22 & 12 & NS \\
Smokers n & 19 & 21 & NS \\
Smoking pack-yrs & $28.8 \pm 17.6$ & $22.4 \pm 14.4$ & NS \\
\hline
\end{tabular}

\#: Chi-squared Student's t-test. mean \pm SD. BMI: body mass index; M: males; F: females; Ns: nonsignificant.

Table 2. - Symptoms, associated respiratory disorders and qualitative lung function in patients with rheumatoid arthritis $(n=100)$ and controls $(n=88)$

\begin{tabular}{lrrl}
\hline & $\begin{array}{c}\text { Cases } \\
\mathrm{n}\end{array}$ & $\begin{array}{c}\text { Controls } \\
\mathrm{n}\end{array}$ & $\mathrm{p}$-value \\
\hline \multicolumn{3}{l}{ Symptoms and pulmonary diseases } \\
Cough & 32 & 14 & 0.01 \\
Expectoration & 19 & 6 & 0.01 \\
Dyspnoea & 48 & 11 & 0.0001 \\
Associated respiratory disease & 26 & 7 & 0.001 \\
Bronchiectasis & 6 & 0 & 0.03 \\
Abnormalities of PFT values & & & \\
Overall obstruction (FEV1/FVC) & 16 & 4 & 0.003 \\
$\begin{array}{l}\text { Distal obstruction (FEF25-75) } \\
\text { Restrictive lung function (TLC) }\end{array}$ & 18 & 4 & 0.001 \\
Transfer coefficient (TL,CO/VA) & 14 & 3 & NS \\
\hline
\end{tabular}

\#: Chi-squared or Fisher's exact test. $\$$ : number of patients with observed value less than threshold (see Methods). PFT: pulmonary function test; FEV1/FVC: ratio of forced expiratory volume in one second to forced vital capacity; FEF25-75: forced expiratory flow between 25 and $75 \%$ of forced vital capacity; TLC: total lung capacity; $T \mathrm{~L}, \mathrm{CO} / V_{\mathrm{A}}$ : transfer coefficient (transfer factor of the lungs for carbon monoxide/alveolar volume); Ns: nonsignificant.
Table 3. - Results of pulmonary function tests in patients with rheumatoid arthritis $(n=85)$ and controls $(n=88)$

\begin{tabular}{lccc}
\hline Lung function & $\begin{array}{c}\text { Cases } \\
\% \text { pred }\end{array}$ & $\begin{array}{c}\text { Controls } \\
\% \text { pred }\end{array}$ & p-value $^{\#}$ \\
\hline VC & $99 \pm 16$ & $106 \pm 17$ & 0.004 \\
TLC & $97 \pm 13$ & $99 \pm 12$ & NS \\
FEV1 & $92 \pm 21$ & $102 \pm 19$ & 0.001 \\
FEV1/FVC & $94 \pm 13$ & $98 \pm 8$ & 0.02 \\
FEF25-75/FVC & $77 \pm 29$ & $84 \pm 22$ & 0.06 \\
FEF25-75 & $78 \pm 32$ & $88 \pm 25$ & 0.03 \\
TL,CO & $102 \pm 21$ & $104 \pm 18$ & NS \\
TL,CO/VA & $114 \pm 19$ & $110 \pm 18$ & NS \\
RV & $102 \pm 27$ & $102 \pm 25$ & NS \\
RV/TLC & $103 \pm 25$ & $100 \pm 18$ & NS \\
\hline
\end{tabular}

\#: Student's t-test or Mann-Whitney test (if unequal variances). Values are presented as mean \pm SD. The pulmonary function test results of three patients with rheumatoid arthritis are omitted as they were not interpretable. TLC: total lung capacity, FEF25-75/FVC: ratio of FEF25-75 to forced vital capacity; TL,CO: single breath transfer factor of the lungs for carbon monoxide; RV: residual volume; RV/TLC: ratio of residual volume to total lung capacity. For further definitions see legend to table 2 .

There was also a significant difference for all the spirometric indices of obstruction (table 3): FEV1, FEV1/ FVC, FEF25-75, FEF25-75/FVC. However, there was no significant difference between the RA and control groups in the parameters assessing restrictive lung values and impairment of the alveolar-capillary barrier ( $T \mathrm{~L}, \mathrm{CO} / \mathrm{alve}-$ olar volume $(V \mathrm{~A}))$.

\section{Comparison of groups after exclusion of smokers $(n=148)$}

There were no differences between the patients with RA $(n=81)$ and controls $(n=67)$ in demographic details (age, gender, body mass index) or previous illness. However, the nonsmoking RA group had more respiratory events $(n=22)$ than did the controls $(n=10)$, although the difference was not significant $(\mathrm{p}=0.07)$.

In table 4 , it can be seen that there was a significant difference for respiratory symptoms and associated pulmonary conditions. The associated respiratory pathologies in the RA group $(n=16)$ included: 4 cases of chronic

Table 4. - Symptoms, associated respiratory disorders and qualitative lung function in nonsmokers with rheumatoid arthritis $(n=81)$ and controls $(n=67)$

\begin{tabular}{lrcc}
\hline & $\begin{array}{c}\text { Cases } \\
\mathrm{n}\end{array}$ & $\begin{array}{c}\text { Controls } \\
\mathrm{n}\end{array}$ & $\mathrm{p}$-value \\
\hline $\begin{array}{l}\text { Symptoms and pulmonary diseases } \\
\text { Cough }\end{array}$ & 23 & 7 & 0.007 \\
Expectoration & 12 & 0 & 0.0005 \\
$\begin{array}{l}\text { Dyspnoea } \\
\text { Associated respiratory disease }\end{array}$ & 37 & 5 & 0.0001 \\
Bronchiectasis & 16 & 2 & 0.001 \\
$\begin{array}{l}\text { Abnormalities of PFT values } \\
\text { Overall obstruction (FEV1/FVC) }\end{array}$ & 11 & 0 & 0.03 \\
Distal obstruction (FEF25-75) & 14 & 0 & 0.0006 \\
Restrictive lung function (CPT) & 6 & 3 & 0.0001 \\
Transfer coefficient $(T \mathrm{~L}, \mathrm{CO} / \mathrm{VA}$ ) & 12 & 7 & $\mathrm{NS}$ \\
\hline
\end{tabular}

\#: Chi-squared or Fisher's exact test. observed value less than threshold (see Methods). For definitions see legend to table 2 . 
bronchitis, 4 asthma, 2 fibroses and 6 bronchiectasis; while in the control group, only two cases of asthma were noted. There was a significant difference in the qualitative indices of obstruction.

PFTs were interpretable in 68 nonsmoking patients and in the 67 nonsmoking controls (table 5). There were no significant differences in FEF25-75 and FEF25-75/ $\mathrm{FVC}$, although FEV1 and FEV1/FVC differed between the two groups. There were no significant differences in total pulmonary capacity or alveolar-capillary transfer.

\section{Explanatory analysis}

For the nonsmoking RA patients $(\mathrm{n}=81), 15$ patients showed signs of obstructive disease, among them 14 had overall obstruction and 11 had peripheral obstruction. Two patients had an associated restrictive disease. The true percentage of obstruction, after exclusion of associated restrictive conditions, was thus 13 out of $81(16 \%)$. No cases of obstruction were noted in the non-smoking controls $(n=67)$.

The qualitative indices of obstruction (overall and distal obstruction) were analysed using the Chi-squared test. No relationship was noted between the functional disorder and the pulmonary or cardiac history, Sjögren's syndrome (Chi-squared by Fisher test=0.08). Similarly, there was no relationship between the types of treatment (gold, methotrexate, corticoids) and the spirometric abnormalities.

A linear regression analysis was carried out on the two main PFT parameters (FEV1/FVC and FEF25-75), with the following variables: body mass index (BMI), age, duration of RA, Ritchie's index, ESR, and cigarette pack-yrs. Two qualitative variables were employed to stratify the analysis: pulmonary or cardiac history. Including smokers and nonsmokers, the only variable in the equation was the number of pack-yrs $(16 \%$ of the explained variance for FEF25-75 and $19 \%$ for FEV1/ FVC). Excluding the smokers, the results, as a function of a history of respiratory disease, are shown in tables 6 and 7. For FEF25-75 (table 6), ESR and duration of RA emerged as significant factors in patients without pulmonary history; duration of RA and BMI in those with pulmonary history. For FEV1/FVC (table 7), Ritchie's index and duration of RA were the two explanatory

Table 5. - Results of pulmonary function tests in nonsmokers (68 out of 81 nonsmoking patients with rheumatoid arthritis had interpretable pulmonary function test results, and all 67 nonsmoking controls)

\begin{tabular}{lccc}
\hline Lung function & $\begin{array}{c}\text { Cases } \\
\% \text { pred }\end{array}$ & $\begin{array}{c}\text { Controls } \\
\% \text { pred }\end{array}$ & p-value $^{\#}$ \\
\hline VC & $100 \pm 16$ & $109 \pm 16$ & 0.002 \\
TLC & $97 \pm 13$ & $99 \pm 13$ & NS \\
FEV1 & $94 \pm 20$ & $106 \pm 16$ & 0.001 \\
FEV1/FVC & $95 \pm 12$ & $100 \pm 7$ & 0.05 \\
FEF25-75 & $82 \pm 32$ & $91 \pm 22$ & NS \\
FEF25-75/FVC & $81 \pm 28$ & $85 \pm 20$ & NS \\
$T$ L,CO & $102 \pm 20$ & $104 \pm 18$ & NS \\
TL,CO/VA & $114 \pm 19$ & $111 \pm 17$ & NS \\
RV & $101 \pm 27$ & $100 \pm 24$ & NS \\
RV/TLC & $103 \pm 24$ & $98 \pm 16$ & NS \\
\hline
\end{tabular}

\#: Student's t-test or Mann-Whitney test (if unequal variances). Values are presented as mean \pm SD. For definitions see legend to tables 2 and 3.
Table 6. - Stepwise multilinear regression for FEF25-75 in nonsmoking patients with rheumatoid arthritis $(n=65)$, according to pulmonary history

\begin{tabular}{lllll}
\hline Variables & $\mathrm{R}^{2}$ & $\mathrm{VIF}$ & Tolerance & $\mathrm{p}$-value \\
\hline Nonsmokers without pulmonary history & & \\
ESR & 0.14 & 1.05 & 0.95 & 0.01 \\
Duration of RA & 0.24 & 1.05 & 0.95 & 0.07 \\
Nonsmokers with pulmonary history & & \\
Duration of RA & 0.25 & 1.08 & 0.93 & 0.04 \\
BMI & 0.38 & 1.07 & 0.93 & 0.09 \\
\hline
\end{tabular}

FEF25-75: forced expiratory flow between 25 and $75 \%$ of forced vital capacity. ESR: erythrocyte sedimentation rate; RA: rheumatoid arthritis; BMI: body mass index; $\mathrm{R}^{2}$ : coefficient of regression; VIF: variance inflation factor. VIF and tolerance are collinearity tests. See Statistical analysis section for explanation of the stepwise regression.

Table 7. - Stepwise multilinear regression for FEV $1 / F V C$ in nonsmoking patients with rheumatoid arthritis $(n=62)$, according to pulmonary history

\begin{tabular}{lllll}
\hline Variables & $\mathrm{R}^{2}$ & $\mathrm{VIF}$ & Tolerance & $\mathrm{p}$-value \\
\hline Nonsmokers without & pulmonary history & & \\
Ritchie's index & 0.15 & 1.02 & 0.98 & 0.05 \\
Duration of RA & 0.20 & 1.02 & 0.99 & 0.19 \\
Nonsmokers with pulmonary history & & \\
Duration of RA & 0.23 & 1.04 & 0.99 & 0.07 \\
BMI & 0.42 & 1.00 & 0.99 & 0.07 \\
Ritchie's index & 0.54 & 1.00 & 0.99 & 0.15 \\
\hline
\end{tabular}

FEV1/FVC: ratio of forced expiratory volume in one second to forced vital capacity. For further definitions see legend to table 6. See Statistical analysis section for explanation of the stepwise regression.

factors in the first group; duration of RA, BMI and Ritchie's index in the second group. In the nonsmokers, the models, stratified as a function of history of heart disease, produced FEF25-75, ESR, BMI, and Ritchie's index as explanatory factors; while for FEV1/FVC, BMI and Ritchie's index emerged as explanatory factors.

There were no significant differences in PFT parameters between the smoking patients with RA and smoking controls (Mann-Whitney's test for quantitative variables and Chi-squared test for the respiratory symptoms).

\section{Discussion}

The present study showed that the number of obstructive syndromes was higher in patients with RA than in patients with other rheumatological conditions. The percentage of associated respiratory disorders, especially bronchiectasis, was also higher in the patients with RA.

\section{Discussion of the method}

There are various sources of bias in case-control studies of this type.

Selection bias. The PFT requirement may have selected certain patients, especially in the control group, which would tend to enhance differences between the two groups.

Self-report bias inherent in studies using questionnaires. These are not sensitive enough, even associated with PFT, to predict isolated radiological abnormalities, which 
may account for the low rates of fibroses reported in studies of this type. This bias is less significant for bronchiectasis, which is often accompanied by symptoms recorded in the history.

Bias related to the PFT procedures. This was minimized in the control group, all of whom had interpretable results, as opposed to only $85 \%$ of the RA patients. The requirement for three reproducible manoeuvres accounts for the decrease in the sample size of the RA patients (in comparison with the initial group). However, there was no difference in principal demographic variables between RA subjects who had interpretable and non-interpretable PFT.

\section{Respiratory conditions occurring during $R A$}

Patients with RA frequently have respiratory disorders including: pleurisy, obstructive pulmonary disease, interstitial lung disease, parenchymatous nodules and, less commonly, bronchiectasis [1,9].

In the present study, the following disorders were found: $13 \%$ chronic bronchitis (including smokers), $9 \%$ pleurisy, $6 \%$ bronchiectasis, $5 \%$ asthma and $2 \%$ fibroses. The $2 \%$ fibroses, although low, is in line with the findings of other studies $[2,11,13]$. RÉMY-JARDIN et al. [19] suggest that the low rates of fibroses in clinical studies is due to the lack of sensitivity of questionnaires and the absence of systematic CT results. They point out that less than $5 \%$ of interstitial pathologies are detected in such studies. The present study is of the same type and suffers from the same sources of bias. Although the incidence of lesions will depend on the diagnostic methods employed, we were interested here in obstructive lesions using the results of lung function tests.

The association between RA and bronchiectasis is still a matter of debate. Lesions of bronchiectasis have been observed on autopsy $[20,21]$, with a prevalence ranging $5.2-12 \%$. These figures are higher than those for the general population [22], for which only a few surveys of the prevalence of bronchiectasis have been reported $(0.13 \%$ in 1953 , and $0.25 \%$ in 1963) [1]. It is likely that this prevalence has dropped since these reports due to more widespread vaccination and early antibiotic treatment. The prevalence in the USA is reported to be 4 per 10,000 [1]. In a clinical and radiological study, WALKER [2] found $3.1 \%$ of bronchiectasis in patients with RA and $0.3 \%$ in patients with degenerative rheumatological conditions.

RÉMY-JARDIN et al. [19], using high resolution computed tomography scan (HRCT), found $21 \%$ of bronchectasis or bronchiolectasis or both. The HRCT scan images detected $8 \%$ abnormalities in asymptomatic patients, which accounts for the differences in results from studies, such as ours, based on clinical and radiological findings. A higher detection sensitivity was found in the patients with symptoms (69\%) than in those without $(29 \%)$. These results highlight the difficulties in comparing studies using different definitions of disease. In a case-control study of this type, the two groups are subjected to the same sources of bias, such as utilization of clinical and radiological findings, nonsystematic CT scan. Nevertheless, we found more cases of bronchiectasis in the RA group.
The pathophysiology of the relationship between RA and bronchiectasis has yet to be elucidated. In 1985, BAMJI and CoOKE [23] noted a significant incidence of respiratory infections in patients with RA, suggestive of an iatrogenic effect of the treatment, which might favour recurrent pulmonary infections. In cases where the bronchiectasis precedes the RA, other mechanisms need to be invoked, such as the formation of immune complexes which may enhance damage to joints [24, 25]. BEGIN et al. [26] found, in the absence of infections, distal bronchial obstructions and a high percentage of neutrophils in the alveolar lavage fluid from patients with RA. They were in favour of an immunological mechanism, in view of the widespread thickening of the bronchial wall.

The link between obstructive lung disease and the severity of rheumatological lesions is also a matter of debate. SHADICK et al. [1] reported more severe rheumatological lesions in 18 patients with RA presenting secondary bronchiectasis, although other authors have failed to make this observation [3, 25].

\section{Spirographic disorders in patients with $R A$}

Comparisons are also hampered by the differences in selection of patients and the fact that the normal ranges for spirometric parameters (lower limits) differ considerably.

Impairments of respiratory function are common in RA patients: in a study of 41 cases of RA, FRANCK et al. [9] found $41.4 \%$ with spirographic abnormalities, including alterations in lung volume and interstitial lung disease. There was no relationship with rheumatoid factor, the duration of the RA, or the stage of the disease.

Prevalence of airway obstructive disorders has seemed to be high in RA diseases. Nouvet et al. [27] found obstructive disorders in $50 \%$ of their cases of RA. Many epidemiological studies have been performed to explain these findings. An early group of case controls studies, recruited controls from the general population. SASSOON et al. [10] found no difference in obstruction between 19 nonsmoking RA patients and 47 control patients taken from the general population. GEDDEs et al. [6] compared 100 cases of RA with 84 patients taken from the general population. They found a significant decrease in FEV1, FEV1/FVC and MMEFR in the smoking RA patients, and a decreased FEV1, VC and MMEFR in the nonsmoking RA patients.

Using a similar design to the present study, with a combination of spirometric data and clinical questionnaire, BANKs et al. [11] found that spirometric parameters (FEV1, VC, MMEFR) were reduced in the nonsmokers and the smokers, although the ratios (FEV1/FVC and MMEFR/VC) were no different from those of control patients. They suggested that the reduction in MMEFR was due to an associated restrictive disorder.

A second group of studies recruited controls with other rheumatological conditions. ColLins et al. [5] compared smokers with RA and other smokers with degenerative rheumatological conditions. They found a reduction in MMEFR in the RA patients, although $77 \%$ of these patients were also smokers. The incidence of obstructive disorders was higher in the smokers with RA than 
in control smokers, and they concluded that RA potentiated the action of tobacco. In a similar study [8], age, height, gender and RA all emerged as independent variables from an explanatory analysis for $V^{\prime} \max , 50$. The authors also found an association between the MSalpha-1 phenotype and the obstructive syndrome. HASSAN et al. [13] carried out a study of similar design to evaluate bronchial hyperreactivity in RA patients. The prevalence of smoking was higher in their series (64\% of current or ex-smokers) than in the present study, but the incidence of fibroses was the same $(2 \%)$. They found a significant difference in the incidence of bronchial hyperreactivity in the RA patients $(55 \%)$ relative to patients with other rheumatological conditions (16\%), after exclusion of the smoking factor. The prevalence of wheezing was $18 \%$ in the RA patients. No correlations with history, treatment, or biological markers were observed in this series, although there was a difference between the RA patients with bronchial hyperactivity and those without. The obstructive disease may, thus, have favoured bronchial hyperactivity in the RA patients.

In the present study, we compared RA patients with patients suffering from rheumatological conditions. The RA patients were all out-patients and their condition was essentially stable; they had a low incidence of pulmonary fibrosis, based on clinical and radiological findings. The control patients suffered mainly from degenerative diseases (arthritis) or infectious conditions, chosen to be as comparable as possible to the RA group. This may account for the lack of difference in restrictive syndrome, which has frequently been found in other similar case-control studies with a control group drawn from the general population. However, we found a greater incidence of obstructive syndrome in the RA patients. This disorder appears to be more specific to RA than to other rheumatological conditions. The small number of patients with bronchiectasis in the present series did not permit a specific analysis, but the bronchiectasis almost certainly contributed to the observed rate of obstructive disorders.

The explanatory analysis needs to be interpreted with caution, as only FEV1/FVC and FEF25-75 could be modelled and the sample size was small. Inclusion of history of pulmonary disease altered the factors accounting for the the obstructive indices. For the nonsmokers with a history of respiratory disorders, the explanatory model for FEF25-75 generated ESR, the duration of the RA and $\mathrm{BMI}$ as explanatory factors. For FEV1/FVC, the duration of the RA, Ritchie's index and BMI emerged. Although we could not unravel the interrelations between lung disorders and RA, our results, along with those of other authors, lend further support to the link between the pulmonary diseases and the severity of the rheumatological lesions.

Various pathophysiological mechanisms have been suggested for the respiratory disorders in RA. They have been attributed to a variety of factors including: underlying bronchial hyperreactivity [13]; abnormalities in distal bronchioles [22]; association with a deficit in alpha ${ }_{1}$-antitrypsin [8]; recurrent respiratory infections [6]; or treatment with penicillamine, which may favour bronchiolitis [7]. Certain authors have failed to find any link between the obstructive syndrome and Sjögren's syndrome [5], whereas others have found more severe disorders in patients with Sjögren's syndrome [26, 28]. The relationships between airway obstructive disorders, pulmonary diseases (such as symptomatic or nonsymptomatic bronchiectasis), and the severity of the RA will need to be established in future epidemiological studies.

In conclusion, our results have indicated a higher number of cases of obstructive syndrome in patients with rheumatoid arthritis compared to controls with other rheumatic conditions. This was accompanied by a higher percentage of associated respiratory disorders, which may be accounted for, at least in part, by the higher occurrence of bronchiectasis. Nevertheless, our findings support those of others studies and argue in favour of a specific obstructive disorder in rheumatoid arthritis. We could not discern whether this was due to the nature of the treatment received by these patients or to a manifestation of the disease outside the joints. Further studies will be required to establish the exact relationship between the obstructive respiratory disorder and rheumatoid arthritis, and to throw further light on the pathophysiological mechanism involved.

\section{References}

1. Shadick NA, Fanta CH, Weinblatt ME, O'Donnell W, Coblyn JS. Bronchiectasis: a late feature of severe rheumatoid arthritis. Medicine 1994; 73: 161-170.

2. Walker WC. Pulmonary infections and rheumatoid arthritis. $Q J$ Med 1967; 36: 239-251.

3. Mc Mahon MJ, Swinson DR, Shettar S, et al. Bronchiectasis and rheumatoid arthritis: a clinical study. Ann Rheum Dis 1993; 52: 776-779.

4. Solanki T, Neville E. Bronchiectasis and rheumatoid disease: is there an association? Br J Rheumatol 1992; 31: 691-693.

5. Collins RL, Turner RA, Myron Johnson A, Whitley NO, McLean R. Obstructive pulmonary disease in rheumatoid arthritis. Arthritis Rheum 1976; 19: 623-628.

6. Geddes DM, Webley M, Emerson PA. Airways obstruction in rheumatoid disease. Ann Rheum Dis 1979; 38: 222-225.

7. Radoux V, Menard HA, Begin R, Decary F, Koopman WJ. Airways disease in rheumatoid arthritis patients. Arthritis Rheum 1987; 30: 249-256.

8. Mountz JD, Turner RA, Collins RL, Gallys KR, Semble EL. Rheumatoid arthritis and small airway function. Arthritis Rheum 1984; 27: 728-736.

9. Franck ST, Weg JG, Harkleroad LE, Fitch RF. Pulmonary dysfunction in rheumatoid disease. Chest 1973; 63: 27-34.

10. Sassoon CSH, Mcalpine SW, Tashkin DP, Baydur A, Quismorio FP, Mongan ES. Small airways function in nonsmokers with rheumatoid arthritis. Arthritis Rheum 1984; 27: 1218-1226.

11. Banks J, Banks C, Cheong B, et al. An epidemiological and clinical investigation of pulmonary function and respiratory symptoms in patients with rheumatoïd arthritis. $Q J$ Med 1992; 85: 795-806.

12. Perez T, Dansin E, Wallaert B, Tonnel AB. Manifestations respiratoires de la polyarthrite rhumatoïde. Rev Mal Respir 1991; 8: 169-189.

13. Hassan WU, Keaney NP, Holland CD, Kelly CA. Bronchial hyperreactivity and airflow obstruction in rheumatoid arthritis. Ann Rheum Dis 1994; 53: 511-514. 
14. Quanjer PhH, Tammeling GJ, Cotes JE, Pedersen OF, Peslin R, Yernault JC. Recommandations européennes pour les explorations fonctionnelles respiratoires: volumes pulmonaires et débits expiratoires forcés. Rev Mal Respir 1994; 11: S5-S40.

15. Organisation Mondiale de la Santé. Questionnaire sur les symptômes respiratoires in OMS éd: le dépistage précoce des maladies professionnelles. 1st éd. Genéve, 1989: pp. 278-283.

16. Ritchie DM, Boyle JA, MacInnes JM. Clinical studies with an articular index for the assessment of joint tenderness in patients with rheumatoid arthritis. $Q \mathrm{~J} \mathrm{Med}$ 1968; 37: 393-406.

17. Bouchet C, Guillemin F, Briancon S. Comparaison de trois instruments de qualité de vie pour l'étude longitudinale de la polyarthrite rhumatoïde. Rev Epidemiol Santé Publique 1995; 43: 250-258.

18. Vitali C, Bombardieri S, Moutsopoulos HM. Preliminary criteria for classification of Sjögren's syndrome. Arthritis Rheum 1993; 36: 340-347.

19. Rémy-Jardin M, Rémy J, Cortet B, Mauri F, Delcambre B. Lung changes in rheumatoid arthritis: CT findings. Radiology 1994; 193: 375-382.

20. Aronoff A, Bywaters EGL, Fearnley GR. Lung lesions in rheumatoid arthritis. Br Med $J$ 1955; 2: 228-233.

21. Brannan HM, Good CA, Divertie MB, Baggenstoss AH.
Pulmonary disease associated with rheumatoid arthritis. JAMA 1964; 189: 914-918.

22. Le Coz A. Polyarthrite rhumatoïde et dilatation des bronches. Medical thesis, Université de Rennes, France, 1990.

23. Bamji A, Cooke N. Rheumatoid arthritis and chronic bronchial suppuration. Scand J Rheumatol 1985; 14: 15-21.

24. Hilton AM, Doyle L. Immunological abnormalities in bronchiectasis with chronic bronchial suppuration. $\mathrm{Br} J$ Dis Chest 1978; 72: 207-216.

25. Kelly C, Gardiner Ph. The relationship between rheumatoid arthritis and bronchiectasis (Letter). Ann Rheum Dis 1994; 53: 482-483.

26. Bégin R, Massé S, Cantin A. Airways diseases in a subset of nonsmoking rheumatoid patients: characterization of the disease and evidence for an autoimmune pathogenesis. Am J Med 1982; 72: 743-750.

27. Nouvet G, Decoux L, Lerebours Pigeonniére C, Thiberville L, Tardif C, Le Loet X. L'exploration fonctionnelle respiratoire au cours de la polyarthrite rhumatoïde: aspect évolutif chez 50 patients (Abstract). Rev Mal Respir 1988; 5: S139.

28. Andonopoulos AP, Constantopoulos SH, Drosos AA, Moutsopoulos HM. Pulmonary function of nonsmoking patients with rheumatoid arthritis in the presence and absence of secondary Sjögren's syndrome: a controlled study. Respiration 1988; 53: 251-258 